\title{
Interactions between HIV and anaemia in an asymptomatic infected population in South Africa
}

\author{
P. A. Aryee ${ }^{1,2}$, R. A. Annan ${ }^{2}$, A. A. Jackson ${ }^{2}$, B. M. Margetts ${ }^{2}$ and E. Vorster ${ }^{3}$ \\ ${ }^{1}$ University for Development Studies, School of Medicine \& Health Sciences, Tamale, Ghana, ${ }^{2}$ University of Southampton, \\ Institute of Human Nutrition, Southampton General Hospital, Southampton, UK and ${ }^{3}$ North-West University, Centre of \\ Excellence for Nutrition, Potchefstroom, South Africa
}

HIV infection is associated with a reduction in CD4+ numbers ${ }^{(1)}$ and altered Fe metabolism ${ }^{(2)}$. Anaemia in HIV infection has a multifactorial aetiology, occurs frequently and is associated with increased disease progression and a shortening of survival time ${ }^{(3)}$. Direct viral effects, reduced energy and nutrient intake, inflammation and metabolic alterations have been proposed as important biological factors enhancing the risk of anaemia with infection. The aim of the present study was to determine the prevalence and predictors of anaemia in individuals infected with HIV.

The THUSA survey, a cross-sectional population-based study in the North West Province of South Africa served as a source of secondary data. A total of 1854 'apparently-healthy' males and females aged $\geq 15$ years were selected, of which 216 (11.9\%) were subsequently found to be HIV seropositive. Energy and micronutrient intake was assessed using a validated quantitative FFQ. Anthropometric and biochemical measurements were undertaken. Fe status and anaemia were assessed using Hb, packed cell volume, serum Fe, serum ferritin, total Fe-binding capacity and percentage saturation. Haematological indices were analysed using univariate ANOVA. The results indicated that $\mathrm{Hb}$, serum $\mathrm{Fe}$, ferritin and total Fe-binding capacity were lower; and percentage saturation was higher but not significant in individuals who were HIV seropositive compared with the non-infected individuals.

Packed cell volume was significantly lower $(P<0.001)$ in the infected subjects. Prevalence of anaemia in the study population was generally high $(46.5 \%)$, but comparatively higher in the infected than the uninfected population $(51.4 \% v .45 .8 \% ; P=0.123)$. Combining serum ferritin and $\mathrm{Hb}$ cut-offs indicated that subjects infected with HIV were more prone to both Fe-deficiency anaemia and anaemia of chronic inflammation. Binary logistic regression analysis showed urban-transitional settlement (OR 2.06 (95\% CI 1.11, 3.85), $P=0.023)$; higher serum total proteins (OR 4.41 (95\% CI 2.7, 7.59), $P<0.001)$; lower serum albumin (OR $2.85(95 \%$ CI $1.67,4.84), P<0.001)$; and higher percentage saturation (OR $1.81(95 \%$ CI $1.11,2.94), P=0.017)$ as significant predictors of anaemia.

It appears that chronic inflammatory processes and altered metabolism in HIV disease influence Fe metabolism and risk of anaemia. HIV may thus be a significant contributor to the morbidities and mortalities associated with anaemia in a population for whom anaemia is a serious public health problem. Efforts at reducing anaemia in the population must be targeted at ameliorating inflammation.

1. Bain BJ (1999) Curr Opin Hematol 6, 89-93.

2. Butensky E, Kennedy CM, Lee MM et al. (2004) J Assoc Nurses AIDS Care 15(6), 31-45.

3. Moyle G (2002) AIDS Rev 4, 13-20. 